\title{
Secondary Science with ROTSE Data
}

\author{
J. Štrobl ${ }^{\mathrm{a}, \mathrm{b}}$, R. Hudec ${ }^{\mathrm{a}}$, M. Jelínek ${ }^{\mathrm{a}}$, V. Šimon ${ }^{\mathrm{a}}$, F. Hroch ${ }^{\mathrm{a}, \mathrm{c}}$, C. Akerlof ${ }^{\mathrm{d}}$, ROTSE \\ team $^{\mathrm{d}}$ \\ ${ }^{a}$ Astronomical Institute Academy of sciences of the Czech Republic, CZ-251 65 Ondřejov, Czech Republic \\ ${ }^{b}$ Astronomical Institute, Charles University, Prague, Czech Republic \\ ${ }^{c}$ Masaryk University, Brno, Czech Republic \\ ${ }^{d}$ University of Michigan, Ann Arbor, Michigan, USA
}

\begin{abstract}
The ROTSE optical GRB follow-up instrument offers an excellent possibility for a secondary science with the data obtained within the sky monitoring. We present and discuss the results of a project of analysing two selected ROTSE monitoring fields with the goal to study the long-term behaviour of the objects located inside. The method developed and tested can be applied in a general way to study light changes of astrophysical objects of various types within the limiting magnitude of the ROTSE device.
\end{abstract}

\section{INTRODUCTION}

The WF (Wide-Field) CCD systems are very commonly used in recent automated observing experiments like ROTSE, LOTIS, BOOTES, BART, TAROT etc. These WF projects come with very special and specific problems, which embarrass the reduction process and following scientific research. This study attempts to find solution of these problems and find nearly comfortable automatic reduction process and test this process on real sky monitoring data from project ROTSE (Akerlof et al. 2000ab, McKay et al. 2000). The main goal of the study was to propose and to test procedures allowing to use the ROTSE monitor data for secondary science such as study of variable astrophysical objects by generating and analyzing their light curves.

\section{WIDE-FIELD CCD IMAGING}

The WF imaging has especially the following problems:

- difficult manipulation with data (one CCD picture has a big size)

- overlapping of stars (high areal density of stars)

- computing speed (really many objects in large FOV)
- problems with calibration (non constant extinction in large FOV)

\section{THE PROCEDURE TESTED}

For the basic reduction the significantly modified software pack "munipack" (http://www.ian.cz/ munipack/) by F. Hroch was used. Most of these modifications should occur in the next version of this software. The other phases of reduction process are realised by a set of quite simple utilities programmed in C. Everything is connected with the small set of shell scripts, which make the use of the whole package easy and comfortable. The whole process can be divided into following steps: (1) The basic (instrumental) calibration (application of dark frame, flat field), (2) The stars identification and photometry process, (3) Matching founded stars with catalogue, (4) Photometric calibration.

First three parts are performed with adapted utilities from package "munipack". The last one (photometric calibration) is based on the calibration of instrumental magnitude regarding to the UBV system by fitting of magnitudes of constant stars to catalogue values.

The reduction process is already functional, though it is still in development.

CP662, Gamma-Ray Burst and Afterglow Astronomy 2001: A Workshop Celebrating the First Year of the HETE Mission,

edited by G. R. Ricker and R. K. Vanderspek

(C) 2003 American Institute of Physics 0-7354-0122-5/03/\$20.00 


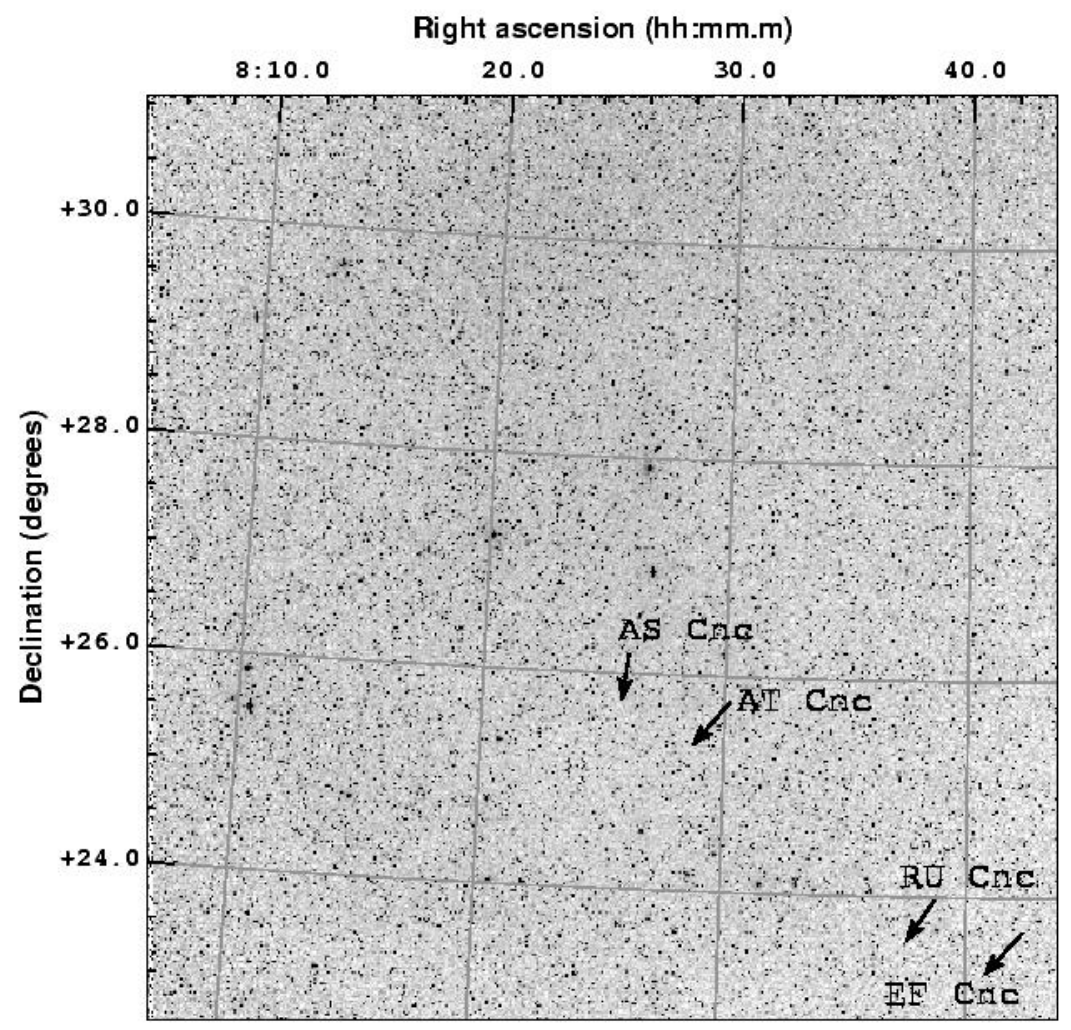

FIGURE 1. The ROTSE field 54b with indicated variable stars whose light curves are shown as examples.

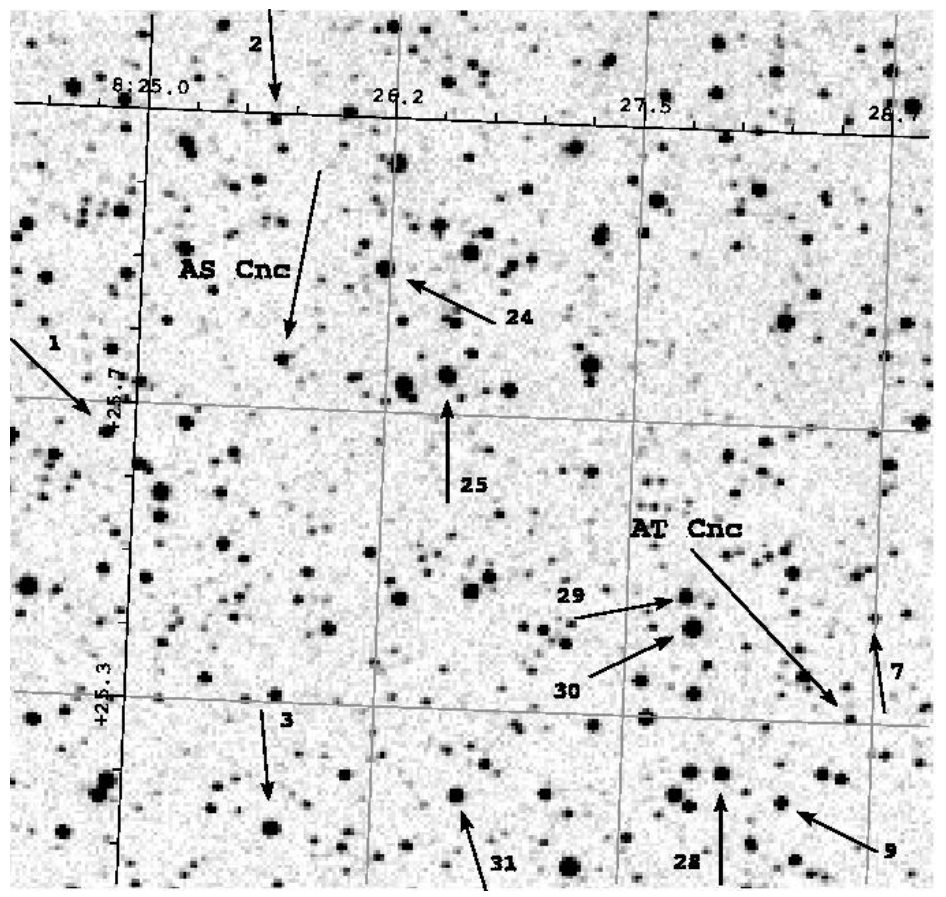

FIGURE 2. The surroudings of stars AS Cnc and AT Cnc on ROTSE CCD image. 

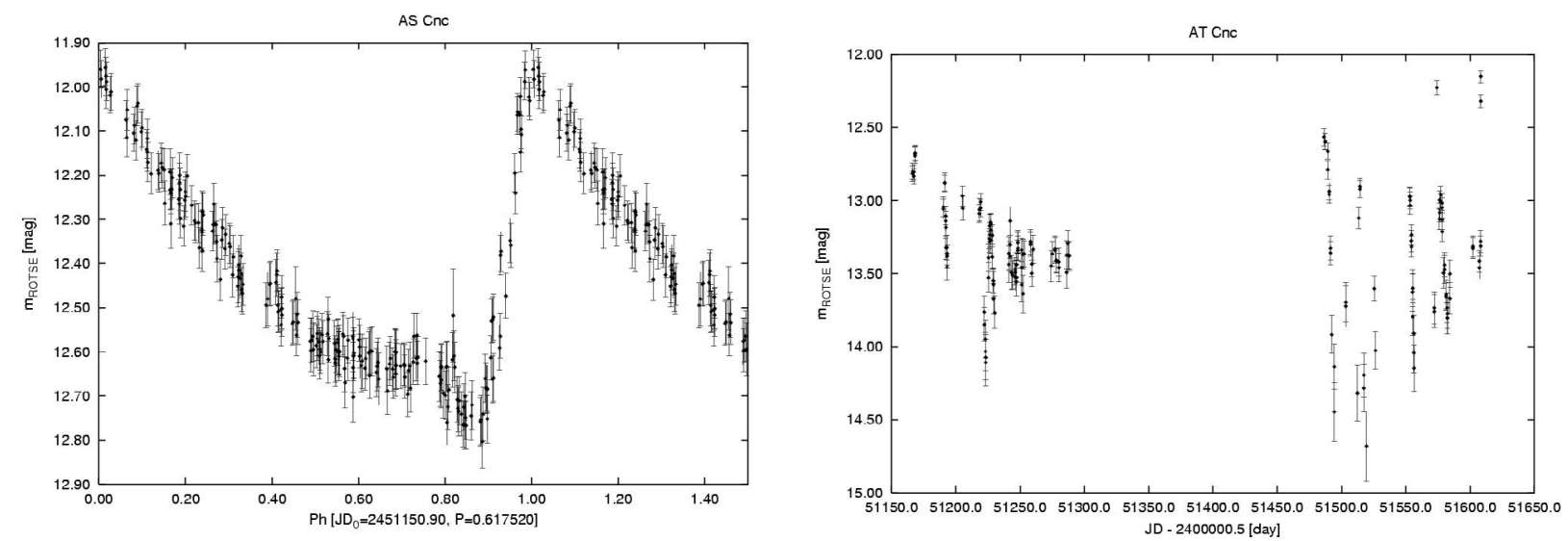

FIGURE 3. Left . The generated light curve (orbital) of AS Cnc. Right. The generated light curve of AT Cnc.

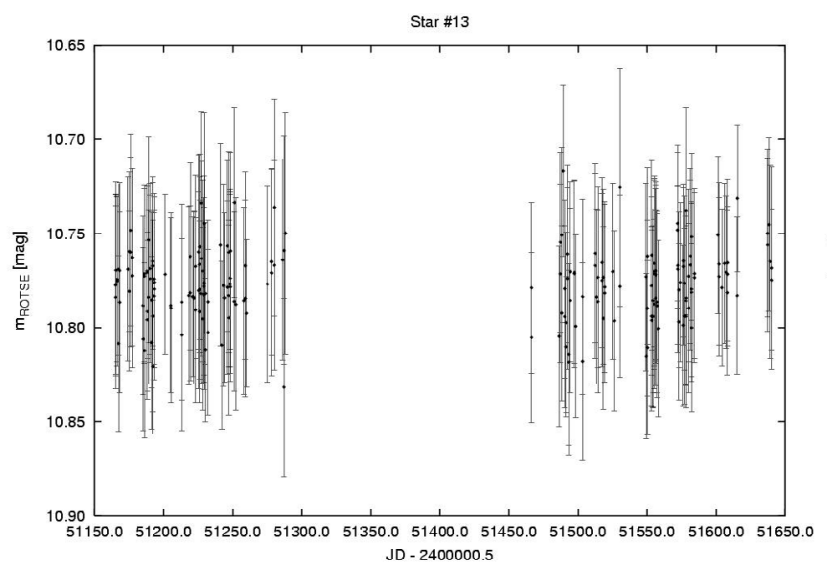

FIGURE 4. The light curves of constant stars

\section{EXAMPLES OF RESULTS}

We present examples of results from processing of the field 54b of the ROTSE survey.

This field has FOV approximately $8 \mathrm{deg}$. Our processing detected about 20000 stars on each picture. The whole process of all data (about 300 pictures) has taken tens of hours (on computer with processor Intel Celeron 566MHz).

\section{CONCLUSION}

The ROTSE WF CCD monitoring data represent a unique database for study of variable astrophysical objects. We have tested procedures dedicated to create light curves for all objects inside the FOV of particular fields. The accuracy of photometric measurements represents few hundreds of magnitude, hence real light changes of order of 0.1 mag can be revealed and analysed.

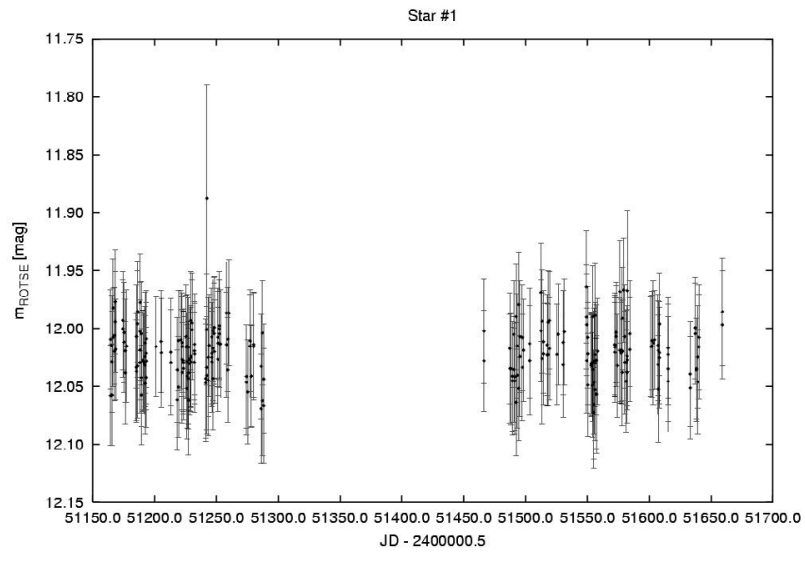

\section{ACKNOWLEDGEMENTS}

This study has been made as Diploma Thesis of Jan Strobl, student of Charles University in Prague.

The project utilizes data obtained by the Robotic Optical Transient Search Experiment. ROTSE is a collaboration of Lawrence Livermore National Lab, Los Alamos National Lab, and the University of Michigan (www.umich.edu/ rotse). The work has been also supported by the grant provided by the Grant Agency of the Czech Republic, 205/99/0145.

\section{REFERENCES}

1. Akelof, C. et al.: www.umich.edu/ rotse

2. Akerlof, C. AJ 119 (2000), 1901A.

3. Akerlof, C. et al.: ApJ 532 (2000), L25.

4. McKay, C. et al.: AASM 197 (2000), 4621. 